\title{
PSEUDO-FINITE HARD INSTANCES FOR A STUDENT-TEACHER GAME WITH A NISAN-WIGDERSON GENERATOR
}

\author{
JAN KRAJÍČEK
}

Faculty of Mathematics and Physics, Charles University in Prague

Sokolovská 83, Prague 8, CZ - 186 75, The Czech Republic

e-mail address: krajicek@karlin.mff.cuni.cz

\begin{abstract}
For an NP $\cap$ coNP function $g$ of the Nisan-Wigderson type and a string $b$ outside its range we consider a two player game on a common input $a$ to the function. One player, a computationally limited Student, tries to find a bit of $g(a)$ that differs from the corresponding bit of $b$. He can query a computationally unlimited Teacher for the witnesses of the values of constantly many bits of $g(a)$. The Student computes the queries from $a$ and from Teacher's answers to his previous queries.

It was proved in [Kra11b] that if $g$ is based on a hard bit of a one-way permutation then no Student computed by a polynomial size circuit can succeed on all $a$. In this paper we give a lower bound on the number of inputs $a$ any such Student must fail on. Using that we show that there is a pseudo-finite set of hard instances on which all uniform students must fail. The hard-core set is defined in a non-standard model of true arithmetic and has applications in a forcing construction from [Kra11a].
\end{abstract}

\section{INTRODUCTION}

Consider a function $g:\{0,1\}^{n} \rightarrow\{0,1\}^{m}$ defined as a Nisan-Wigderson generator based on some Boolean function $f$, cf. [NW94]. That is, there is a set system $\left\{J_{i} \subseteq[n]\right\}_{i \in[m]}$ such that

- $\left|J_{i}\right|=\ell$, for all $i$

- $\left|J_{i} \cap J_{j}\right| \leq d$, for all $i \neq j$

and the $i$-th bit of $g(x)$ equals to $f\left(x\left(J_{i}\right)\right)$ where $f:\{0,1\}^{\ell} \rightarrow\{0,1\}$ and $x\left(J_{i}\right)$ is the $\ell$-bit string $x_{j_{1}} \ldots x_{j_{\ell}}$ if

$$
J_{i}=\left\{j_{1}<\cdots<j_{\ell}\right\} .
$$

We are interested in the case when $f$ is a hard bit $B(v)$ of a polynomial-time computable one-way permutation $h:\{0,1\}^{\ell} \rightarrow\{0,1\}^{\ell}$ :

$$
f(u):=B\left(h^{(-1)}(u)\right) .
$$

1998 ACM Subject Classification: F.2.2, F.4.1.

Key words and phrases: Nisan-Wigderson generator, interactive computation, hard core, model theory.

Supported in part by grant IAA100190902. Also partially affiliated with the Institute of Mathematics of the Academy of Sciences.

넴
DOI:10.2168/LMCS-8 (3:09) 2012

(C) J. Krajíček (C) Creative Commons 
If $m>n$ there are strings $b \in\{0,1\}^{m} \backslash R n g(g)$ and with any such string $b$ we associate the following game mathcal $G_{b}$. The game is played by two players, a Student and a Teacher, both knowing $b$. They receive a common input: any $a \in\{0,1\}^{n}$. The Student tries to find $i \in[m]$ such that $g(a)_{i} \neq b_{i}$, certifying thus that $b$ is outside of the range of $g$. The Student will be computationally limited and, in particular, while he will be able to compute permutation $h$ he will not be able to compute function $f$ and to find a suitable bit $i$ himself. Instead he will compute some candidate solution $i_{1}$ and hand it to the Teacher. She has an unlimited computational power and will reply to the Student with the unique $v^{1} \in\{0,1\}^{\ell}$ such that $h\left(v^{1}\right)=a\left(J_{i_{1}}\right)$.

If $B\left(v^{1}\right) \neq b_{i_{1}}$ the game stops with the Student solving the task. Otherwise he computes his next candidate solution $i_{2} \in[m]$ and hands it to the Teacher, gets back $v^{2}$ such that $h\left(v^{2}\right)=a\left(J_{i_{2}}\right)$, etc.

In general the Student will be allowed to present up to $c$ candidate solutions, $c$ some parameter. If he does not find a solution, we say he failed. The Student can be modelled by $c$ functions

$$
S_{1}(x), S_{2}\left(x, y^{1}\right), \ldots, S_{c}\left(x, y^{1}, \ldots, y^{c-1}\right)
$$

computing his candidate solutions:

$$
i_{k}:=S_{k}\left(a, v^{1}, \ldots, v^{k-1}\right), k \leq c
$$

where $v^{j}$ 's are the Teacher's replies.

We are interested in the case when the Student is a small circuit, i.e. the functions $S_{k}$ are computed by circuits of a small total size. The Teacher's moves are uniquely determined. This Student-Teacher way of computing a function grew out of a use of Herbrand's theorem in bounded arithmetic; the Student-Teacher formalism was introduced in [KPS90] (see there also for an overview in a complexity-theoretic language).

Assuming that $h$ is indeed a one-way permutation it was proved in Kra11b, Kra11a that for any fixed $c \geq 1$ no $\mathrm{P} /$ poly Student can solve the task on all inputs $a \in\{0,1\}^{n}$, for $n>>0$. In this paper we are interested in the question whether there exists a set of hard instances $H \subseteq\{0,1\}^{n}$ such that every $\mathrm{P} /$ poly Student fails on most $a \in H$.

This question is motivated by a research in proof complexity; in particular by a conjecture 1 that functions like $g$ are hard proof complexity generators and by a model-theoretic approach to it based on forcing. This proof complexity motivation is discussed in detail in [Kra11a, Part VIII] and in [Kra11b] and we shall not review it here.

In this paper we show that we can combine the lower bound argument from Kra11a, Chpt.31] and [Kra11b] with the model-theoretic set-up of forcing with random variables to give a partial affirmative answer to the question about the existence of sets $H$. The qualification "partial" means that we construct such a set of hard examples as a pseudo-finite set of strings of a non-standard length $n$ in a model of true arithmetic and that the instances from it are hard for uniform students, i.e. the functions $S_{1}(x), S_{2}\left(x, y^{1}\right), \ldots, S_{c}\left(x, y^{1}, \ldots, y^{c-1}\right)$ defining their moves are computed by uniform algorithms. In fact, we can allow the uniform

\footnotetext{
${ }^{1}$ Statement (S) in [Kra11a, Sec.31.4] or in Kra11b], modifying Razborov's Conjecture 2 from Raz??] and related also to Rudich's demi-bit conjecture from Rud97.
} 
students to use common advice strings of all lengths. This partial solution is perfectly adequate for the purpose of the construction in Kra11a, Chpt.31]2 but it would be desirable to have such hard-core sets for non-uniform students as well. This is because the forcing models constructed in that case have some nice properties (in particular, witnessing of quantifiers [Kra11a, Chpt.3] or saturation properties [Kra??]) that may be useful for further research.

The paper is self-contained in the sense that the reader will be able to fully understand the problem and its solution. However, to appreciate its relevance to the forcing construction and subsequently for the proof complexity conjecture alluded to above one needs to consult [Kra11a, Part VIII] or [Kra11b (better both).

The paper is organized as follows. In Section 1 we give details of the problem and use the argument from [Kra11b, Kra11a] to derive a lower bound on the number of inputs every $\mathrm{P} /$ poly student must fail on. In Section 2 some model theory and forcing with random variables is briefly reviewed and the pseudo-finite hard-core set is constructed in Section 3 .

Relevant background can be found in [Kra95, Kra11a, Kra11b. In particular, readers not familiar with non-standard models can find a self-contained introduction to the topic in the appendix in Kra11a.

\section{HARDNESS OF THE GAME}

We shall fix the following parameters in the definition of function $g$ :

$$
m:=n+1, \quad \ell:=n^{1 / 3}, \quad d:=\log (m) .
$$

In applications of Nisan-Wigderson generators $m$ is usually exponentially large but for the purpose of proof complexity the best choice is to have $m$ as small as possible. By [NW94] there is a set system $\left\{J_{i}\right\}_{i}$ with the required properties, and we fix any one of them.

Let $h$ be a polynomial time permutation (we are interested in its restriction to $\{0,1\}^{\ell}$ ) that is one-way and let $B(v)$ be its hard bit. In particular, by this hardness assumption we mean that

- For any fixed $k \geq 1$ no $\mathrm{P} /$ poly algorithm can compute from $u \in\{0,1\}^{\ell}$ the value $B\left(h^{(-1)}(u)\right)$ with the advantage better than $\ell^{-k}$ over $1 / 2$.

Let us fix a string $b \in\{0,1\}^{m} \backslash R n g(g)$. In [Kra11b] (a similar argument is used in [Kra11a, Sec.31.2] for a different purpose) it was proved that no $\mathrm{P} /$ poly Student can succeed in the game mathcal $G_{b}$ on all inputs, assuming that $h$ is indeed one-way. We are now going to sketch the argument in order to extend it a bit and to deduce a lower bound on the number of inputs on which a Student must fail. Any details of the original argument missing here can be found in the proof of [Kra11b, Thm.3.2].

Consider a Student that attempts to succeed in the game mathcal $G_{b}$ on as many inputs $a$ as possible and assume he can ask at most $c$ queries to the Teacher. The lower bound will depend on $c$.

Denote by $W \subseteq\{0,1\}^{n}$ the set of all $a \in\{0,1\}^{n}$ on which the Student succeeds. For $a \in\{0,1\}^{n}$ let us denote by $v^{i}(a)$ the preimage of $a\left(J_{i}\right)$ in $h$, for $i \in[m]$.

\footnotetext{
${ }^{2}$ The original construction was based on an unsuitable sample space as pointed out by S. Buss. This is explained in the last section.
} 
If the Student succeeded on $a$ and $i$ was its last query to the Teacher it means that

$$
f\left(a\left(J_{i}\right)\right) \neq b_{i} \text {. }
$$

Intuitively this gives us some information about the function $f$ as we can deduce its value on the string $a\left(J_{i}\right)$ while receiving during the computation from the Teacher only strings that have little to do with the string $h^{(-1)}\left(a\left(J_{i}\right)\right)$ we would need in order to compute $f$ ourselves. The formal argument makes this intuition precise.

Assume that the Student asked $k$ queries: the candidate solutions the Student produced were $i_{1}, \ldots, i_{k}$ and the last one $i_{k}$ was correct. Call the $k$-tuple $\left(i_{1}, \ldots, i_{k}\right)$ the trace of the computation on $a$. In particular, $k \leq c$. As the witnesses are unique the trace determines also the Teacher's replies. A simple counting argument yields (cf. the proof of [Kra11b, Thm.3.2])

Claim 1: There is a $k$-tuple $\left(i_{1}, \ldots, i_{k}\right) \in[m]^{k}$ for some $k \leq c$ that is the trace of computations on at least a fraction of $\frac{2}{(3 m)^{k}}$ of all inputs from $W$.

Fixing a trace $\bar{i}=\left(i_{1}, \ldots, i_{k}\right)$ satisfying the claim, define for any $u \in\{0,1\}^{\ell}$ and $v \in\{0,1\}^{n-\ell}$ the string $a(u, v) \in\{0,1\}^{n}$ as follows: put bits of $u$ into the positions $J_{i_{k}}$ and then fill the remaining $n-\ell$ positions by bits of $v$. The following claim follows from the proof of Claim 1 by averaging.

Claim 2: There is $e \in\{0,1\}^{n-\ell}$ s.t. at least a fraction of $\frac{1}{(3 m)^{k}}$ more $u \in\{0,1\}^{\ell}$ yield sample $a(u, e) \in W$ whose trace is exactly $\bar{i}$ than those $u$ which yield $a(u, e) \in W$ whose trace properly contains $\bar{i}$.

Fix one such an $(n-\ell)$-tuple $e$. Call any $u \in\{0,1\}^{\ell} \operatorname{good}$ if $a(u, e) \in W$.

The property that two distinct sets from the set system $\left\{J_{i}\right\}_{i}$ intersect in at most $\log (m)$ positions implies that there are, for any row $i \neq i_{k}$, at most $m$ assignments $w$ to bits in $J_{i}$ not set by $e$. Any such $w$ determines, together with $e$, an assignment to variables in $J_{i}$ and hence a string in $\{0,1\}^{\ell}$; denote it $z_{w}$. Let $Y_{i}$ be the set of all preimages of all $z_{w}$ in the permutation $h$. The total bit size of all $Y_{i}$ together is $m^{O(1)}$.

This situation allows us to define an algorithm $C$ that uses as advice the set system $\left\{J_{i}\right\}_{i}$, the string $b$, the trace $\bar{i}$, the partial assignment $e$, and all $m-1$ sets $Y_{i}$. The total size of the advice is again bounded above by $m^{O(1)}$.

The algorithm $C$ attempts to compute the function $f$ on inputs $u \in\{0,1\}^{\ell}$. Let $U$ be those inputs $u \in\{0,1\}^{\ell}$ for which the trace of $\left.a(u, e)\right)$ either equals to $\bar{i}$ or starts with $\bar{i}$, and let $b_{0}$ be the majority value of $f$ on the complement of $U$.

On input $u \in\{0,1\}^{\ell} C$ simulates the Student's computation on the string $a:=a(u, e) \in$ $\{0,1\}^{n}$. If any of the candidate solutions produced in the $j$-th query, $j=1, \ldots, k$, to the Teacher differs from the $j$-th entry in the trace $\bar{i} C$ halts and outputs $b_{0}$.

Otherwise, if the trace of the computation follows $\bar{i}, C$ uses sets $Y_{i}$ to simulate Teacher's replies (these are unique and can be tested as correct). If the computation evolved according to the trace $\bar{i}$ and reached the $k$-th step $C$ outputs $1-b_{i_{k}}$.

The algorithm $C$ outputs the bit $b_{0}$ in all cases except when the computation follows the trace $\bar{i}$ and reaches the $k$-th step. If the computation of the Student were to actually stop at that point then the value $1-b_{i_{k}}$ is indeed equal to $f(u)$. 
Otherwise, if the computation were to continue, we do not have a way to deduce the true value of $f(u)$. The influence of this case can be, however, bounded. By the choice of $e$ in Claim 2 the former case happens for at least a fraction of $\frac{1}{(3 m)^{k}}$ more of all good inputs $u \in\{0,1\}^{\ell}$ than the latter one. If $W=\{0,1\}^{n}$ (as in Kra11b) we would be done: All $u$ are good and as $b_{0}$ is the correct value of $f$ for at least half of $u \notin U$, the algorithm $C$ would compute $f$ with an advantage over $1 / 2$ at least $\frac{1}{(3 m)^{k}}$.

If $W$ is a proper subset of $\{0,1\}^{n}$ this argument fails as we have no control over the number of bad $u$ (i.e. for which $a(u, e) \notin W)$ but the trace of $a(u, e)$ contains $\bar{i}$, i.e. of the size of the set $U \backslash W$. However, if we knew that the size of the complement of $W$ in $\{0,1\}^{n}$ is at most, say:

$$
s_{c}:=\frac{1}{2} \cdot 2^{n^{1 / 3}} \cdot \frac{1}{(3 m)^{c}}
$$

then the above argument works: $s_{c}$ bounds, in particular, the number of bad $u$ and the algorithm $C$ gets the advantage at least

$$
\frac{1}{(3 m)^{k}}-\frac{1}{2} \cdot \frac{1}{(3 m)^{c}} \geq \frac{1}{2} \cdot \frac{1}{(3 m)^{k}} \geq \frac{1}{2} \cdot \frac{1}{(3 m)^{c}} .
$$

The algorithm $C$ needs the same time as the Student except when it simulates a reply of the Teacher and looks for an appropriate witness in one of the sets $Y_{i}$. This is done at most $(c-1)$-times and takes $m^{O(1)}$ time per one witness-search. Hence if the Student is $P / p o l y$ the total time $C$ uses is $c \cdot m^{O(1)}$.

We conclude that assuming that a $\mathrm{P} /$ poly Student fails on less than $s_{c}$ inputs contradicts the hypothesis that $h$ is a one-way permutation. Hence the following statement was established.

Lemma 1.1. Assume that the parameters $n, m, \ell$, the set system $\left\{J_{i}\right\}_{i}$ and the string $b$ satisfy the conditions imposed on them earlier. Assume also that $h$ is a polynomial-time one-way permutation, that $B(v)$ is its hard bit and that $f(u)=B\left(h^{(-1)}(u)\right)$.

Let $c \geq 1$ be arbitrary. Then for any fixed $k \geq 1$, for any $n$ large enough any Student asking at most $c$ queries to the Teacher and computed by circuits of the total size $m^{k}$ must fail on at least

inputs $a \in\{0,1\}^{n}$.

$$
s_{c}:=\frac{1}{2} \cdot 2^{n^{1 / 3}} \cdot \frac{1}{(3 m)^{c}}
$$

Let us remark that if the hardness of the permutation $h$ were exponential, in the sense that for some $\epsilon>0$ a circuit needs to have the size at least $2^{\ell^{\epsilon}}$ in order to compute the hard bit with an advantage at least $2^{-\ell^{\epsilon}}$, then we could allow also exponentially large Students posing up to $n^{\delta}$ queries for some small $\delta>0$ (depending on $\epsilon$ ) and still get a meaningful lower bound. Also, the whole situation can be specialized to various circuit subclasses of $\mathrm{P} /$ poly such as $A C^{0}$ or $N C^{1}$, as discussed in Kra11b. 


\section{SOME MODEL THEORY}

In this section we briefly recall the set-up of forcing with random variables, enough to formulate and prove our result in the next section. However, we shall not go into the details specific for the construction in [Kra11a, Chpt.31] motivating this paper.

Forcing of random variables is a method how to construct models of arithmetic. A special emphasis is given to bounded arithmetic because of its relation to proof complexity but the method is not limited to this theory. The models are formed from random variables on a pseudo-finite sample space and are Boolean-valued.

Let mathcal $M$ be a non-standard $\aleph_{1}$-saturated model of true arithmetic in some language $L$ containing the language of Peano arithmetic and having a canonical interpretation in the standard model. Let $\Omega \in$ mathcal $M$ be an infinite set; as it is an element of the model it is finite from the point of view of mathcal $M$. Let $F \subseteq$ mathcalM be any - not necessarily definable - family of functions $\alpha: \Omega \rightarrow$ mathcal $M$.

The family $F$ will be the universe of a Boolean-valued $L$-structure $K(F)$. The symbols of $L$ are interpreted by composition with functions from $F$. For example, for a $k$-ary function symbol $f$ and any $\alpha_{1}, \ldots, \alpha_{k} \in F$ define the function $f\left(\alpha_{1}, \ldots, \alpha_{k}\right)$ by

$$
f\left(\alpha_{1}, \ldots, \alpha_{k}\right)(\omega):=f\left(\alpha_{1}(\omega), \ldots, \alpha_{k}(\omega)\right), \text { for } \omega \in \Omega .
$$

We need to assume that this function is also in $F$, i.e. that $F$ is $L$-closed in the terminology of Kra11a.

Every atomic $L(F)$-sentence $A$ is naturally assigned a subset $\langle\langle A\rangle\rangle \subseteq \Omega$ consisting of those samples $\omega \in \Omega$ for which $A$ is true in mathcalM.

Combining the idea of Loeb's measure with some measure theory it was shown in Kra11a, Sec.1.2] that if we factor the Boolean algebra of mathcal $M$-definable subsets of $\Omega$ by the ideal of sets of an infinitesimal counting measure we get a complete Boolean algebra mathcalB.

The image of $\langle\langle A\rangle\rangle$ in mathcalB in this quotient is denoted $\llbracket A \rrbracket$. Following Boole [Boo47] and Rasiowa-Sikorski [RS53] this determines the truth value $\llbracket A \rrbracket \in$ mathcalB for any $L(F)$-sentence $A$ : $\llbracket \ldots \rrbracket$ commutes with Boolean connectives and

$$
\llbracket \exists x A(x) \rrbracket:=\bigvee_{\alpha \in F} \llbracket A(\alpha) \rrbracket
$$

and analogously for the universal quantifier.

There are various generalizations of this basic set-up and, in particular, the random variables from the family $F$ can be only partially defined on the sample space $\Omega$, as long as their regions of undefinability have infinitesimal counting measures.

In the particular construction in Kra11a, Chpt.31] the sample space is simply the set $\{0,1\}^{n}$ for some non-standard $n \in$ mathcal $M$, and the family $F$ consisted of partial random variables computed by students operating similarly as in the game mathcal $G_{b}$. More precisely, any partial function $\alpha \in F$ is computed by a $\mathrm{P} /$ poly Student who

- gets an input $\omega \in\{0,1\}^{n}$,

- there is a standard parameter $c$ such that the Student can ask the Teacher for the values of $h^{(-1)}$ on $\omega\left(J_{i}\right)$ for up to $c$ values of $i \in[m]$ ( $c$ is common to all inputs $\omega$ but may differ for different $\alpha$ ),

- if the Teacher's answer $v$ to any query about $h^{(-1)}\left(\omega\left(J_{i}\right)\right)$ does not satisfy $B(v)=b_{i}$ the computation is aborted and $\alpha$ is undefined, 
- if the computation is not aborted after any Teacher's answer the Student outputs at the end an element of mathcalM (necessarily of size polynomial in $n$ ).

Unfortunately such a function $\alpha$ is typically undefined on a (standard) positive fraction 3 on $\{0,1\}^{n}$ and hence the pair $\{0,1\}^{n}, F$ does not conform to the set-up of the construction.

What is needed is an infinite subset $H \subseteq \Omega$, definable in mathcal $M$, such that every student-computed $\alpha$ is defined on all but an infinitesimal fraction of $H$.

In the next section we shall construct such $H$ for uniform students that may all use some common advice string. This is perfectly sufficient for the intended applications of the model. But it would still be desirable to have such a hard-core set for non-uniform students of some superpolynomial size. The reason is that a family $F$ based on such students can be modified into a compact one (in the sense of [Kra11a, Sec.3.4]) and the compactness of $F$ implies that the resulting model $K(F)$ has some nice model-theoretic properties mentioned in the introduction. For example, non-uniform students of sub-exponential size (i.e. the functions $S_{1}(x), S_{2}\left(x, y^{1}\right), \ldots, S_{c}\left(x, y^{1}, \ldots, y^{c-1}\right)$ defining their moves are computed by circuits of total size $2^{n^{o(1)}}$ ) define family $F$ that is already compact.

\section{A construction of a hard-CORE SET}

We assume that a non-standard $n \in$ mathcal $M$, a set system $\left\{J_{i}\right\}_{i}$ with the required properties, a permutation $h$ and its hard bit $B(v)$, and some string $b \in\{0,1\}^{m} \backslash R n g(g)$ are fixed.

Let $w \in$ mathcal $M$ be any string of size polynomial in $n$ and let $F_{w}^{u n i f} \subseteq$ mathcal $M$ be the family of partial random variables on $\{0,1\}^{n}$ defined as $F$ in Section 2 but allowing all Students computing the random variables to use as an advice only the triple $\left(\left\{J_{i}\right\}_{i}, b, w\right)$. This is perfectly sufficient for any application 4 of the eventual model in Sections 31.3. and 31.4 of Kra11a ( $w$ can contain e.g. a proof of a propositional formula or a witness of the membership of $b$ in an NP set, etc.) and has the great advantage that the family $F_{w}^{u n i f}$ is now countable.

Theorem 3.1. There exists an infinite set $H \subseteq\{0,1\}^{n}, H \in$ mathcal $M$, such that each $\alpha \in F_{w}^{u n i f}$ is defined on all samples from $H$.

Proof. Enumerate as $\alpha_{1}, \alpha_{2}, \ldots$ the set $F_{w}^{\text {unif }}$ in such a way that the Student defining $\alpha_{k}$ runs in time $\leq m^{k}$ and asks at most $k$ queries, for all $k \geq 1$.

By the $\aleph_{1}$-saturation there exists a sequence in mathcal $M$ of a non-standard length $t$ whose $k$-th element is $\alpha_{k}$, for all standard $k$ (see [Kra11a, p.9]). We shall denote it suggestively $\left(\alpha_{i}\right)_{i<t}$.

If we take $\alpha_{1}, \ldots, \alpha_{k}$ we can compose the Students defining them by first computing $\alpha_{1}$, if it is not aborted then instead of outputting a value computing $\alpha_{2}$, etc. , and outputting (arbitrary) values only at the end, if the computation is not aborted earlier. The resulting function is computed in time $O\left(k m^{k}\right)$ using at most $k(k+1) / 2 \leq k^{2}$ queries. Hence by Lemma 1.1 it is defined on at least $s_{k^{2}}$ samples from $\{0,1\}^{n}$. This yields the following

\footnotetext{
${ }^{3}$ Contrary to what was claimed in Kra11a L.31.2.1] - I am indebted to S. Buss for pointing it out. See http://www.karlin.mff.cuni.cz/ $\mathrm{krajicek/k2-upravy.html} \mathrm{for} \mathrm{an} \mathrm{explanation} \mathrm{and} \mathrm{a} \mathrm{correction.}$

${ }^{4}$ Note that Kra11b already provided an alternative construction with the same consequences as those described in Kra11a.
} 
Claim: For each standard $k \geq 1$ there exists definable subset $H^{k} \subseteq\{0,1\}^{n}$ of size at least $s_{k^{2}}$ such that all $\alpha_{1}, \ldots, \alpha_{k}$ are defined on all samples from $H^{k}$.

By the Overspill the statement of the Claim holds also for the sequence $\left(\alpha_{i}\right)_{i \leq r}$ for some non-standard $r \leq t$, and we can take $r$ small enough (but still non-standard) such that $s_{r^{2}}$ is non-standard and hence the set $H:=H^{r}$ satisfies the statement of the theorem.

Let us remark in conclusion that proofs of the Boolean case hard-core set theorem of Impagliazzo Imp95 do not seem to work here. This is because $n$ small Students cannot be combined into a one somewhat larger as this would blow-up the number of queries posed to the Teacher.

\section{ACKNOWLEDGMENT}

I am indebted to the two anonymous referees for comments and suggestions.

\section{REFERENCES}

[Boo47] G. Boole. The mathematical analysis of logic. Barclay and Macmillan, Cambridge, 1847.

[Imp95] R. Impagliazzo. Hard-Core Distributions for Somewhat Hard Problems. 36th Annual Symp. on Foundations of Computer Science, Milwaukee, Wisconsin, 23-25 October 1995, IEEE Computer Society, 538-545, 1995.

[Kra95] J. Krajíček. Bounded arithmetic, propositional logic, and complexity theory. Encyclopedia of Mathematics and Its Applications, Cambridge University Press, Vol.60, 1995.

[Kra11a] J. Krajíček. Forcing with random variables and proof complexity. London Mathematical Society Lecture Notes Series, Cambridge University Press, Vol.382, 2011.

[Kra11b] J. Krajíček. On the proof complexity of the Nisan-Wigderson generator based on a hard NP $\cap$ coNP function. J. Mathematical Logic, 11(1): 11-27, 2011.

[Kra??] J. Krajičcek. A saturation property of structures obtained by forcing with a compact family of random variables. submitted preprint, 2012.

[KPS90] J. Krajíček, P. Pudlák, and J. Sgall. Interactive Computations of Optimal Solutions. in: B. Rovan (ed.): Mathematical Foundations of Computer Science (B. Bystrica, August '90), Lecture Notes in Computer Science, Springer-Verlag, 452:48-60, 1990.

[NW94] N. Nisan and A. Wigderson. Hardness vs. randomness. J. Comput. System Sci., 49:149-167, 1994.

[RS53] H. Rasiowa and R. Sikorski. Algebraic treatment of the notion of satisfiability. Fundamenta Mathematicae, 40:62-65, 1953..

[Raz??] A. A. Razborov. Pseudorandom generators hard for $k$-DNF resolution and polynomial calculus resolution. unpublished preprint, 2003.

[Rud97] S. Rudich. Super-bits, demi-bits, and NP/qpoly-natural proofs. in: Proc. of the 1st Int.Symp. on Randomization and Approximation Techniques in Computer Science, LN in Comp.Sci., SpringerVerlag, 1269:85-93, 1997. 